\title{
Erratum: Efficient approach for modeling phonon transmission probability in nanoscale interfacial thermal transport [Phys. Rev. B 91, 174302 (2015)]
}

\author{
Zhun-Yong Ong and Gang Zhang \\ (Received 17 December 2015; published 5 January 2016)
}

DOI: 10.1103/PhysRevB.93.019901

We have discovered typographical errors in two of the key equations, (9) and (13), of our paper. Equation (9), which gives the Caroli formula for the phonon transmission function, should read as

$$
\Xi(\omega)=\operatorname{Tr}\left(\boldsymbol{\Gamma}_{R} \boldsymbol{G}_{C}^{\mathrm{adv}} \boldsymbol{\Gamma}_{L} \boldsymbol{G}_{C}^{\mathrm{ret}}\right) .
$$

Equation (13), which gives the formula for the $t$ matrix, should read as

$$
\boldsymbol{t}=\frac{2 i \omega}{\sqrt{a_{L} a_{R}}} \boldsymbol{V}_{R}(+)^{1 / 2} \boldsymbol{U}_{R}^{\mathrm{ret}}(+)^{-1} \mathcal{G}^{\mathrm{ret}}\left[\boldsymbol{U}_{L}^{\mathrm{adv}}(-)^{\dagger}\right]^{-1} \boldsymbol{V}_{L}(+)^{1 / 2},
$$

where

$$
\mathcal{G}^{\mathrm{ret}}=\boldsymbol{g}_{R}^{\mathrm{ret}}(\omega) \boldsymbol{H}_{R C} \boldsymbol{G}_{C}^{\mathrm{ret}}(\omega) \boldsymbol{H}_{C L} \boldsymbol{g}_{L}^{\mathrm{ret}}(\omega) .
$$

The errors in Eqs. (9) and (13) are typographical in nature and have no bearing on any of the results reported in the paper. Other equations in the paper making use of Eqs. (9) and (13) are unaffected.

We thank Dr. Takuma Shiga of the University of Tokyo for bringing these errors to our attention. 